\title{
Case Tool: Fast Interconnections with New 3-Disjoint Paths MIN Simulation Module
}

\author{
Ravi Rastogi, Amit Singh \\ and Nikhil Singhal \\ Department of CSE and IT, \\ Jaypee University of \\ Information Technology, \\ Waknaghat, Solan-173234, \\ Himachal Pradesh, India
}

\author{
Nitin \\ Department of Computer \\ Science, The Peter Kiewit \\ Institute, College of \\ Information Science and \\ Technology, University of \\ Nebraska at Omaha, Omaha- \\ 68182-0116, Nebraska, \\ United States of America
}

\author{
Durg Singh Chauhan \\ Uttarakhand Technical \\ University, Post Office \\ Chandanwadi, Prem Nagar, \\ Sudohwala, Dehradun- \\ 248007, Uttarakhand, India
}

\begin{abstract}
Multi-stage interconnection networks (MIN) can be designed to achieve fault tolerance and collision solving by providing a set of disjoint paths. In this paper, we are discussing the new simulator added to the tool designed for developing faulttolerant MINs. The designed tool is one of its own kind and will help the user in developing 2 and 3-disjoint path networks. The java technology has been used to design the tool and have been tested on different software platform.
\end{abstract}

\section{Keywords}

Multi-stage Interconnection Networks, Fault-tolerance, 3Disjoint Paths.

\section{INTRODUCTION AND MOTIVATION}

In a multiprocessor system, many processors and memory modules are tightly coupled together with an interconnection network. A properly designed interconnection network certainly improves the performance of such multiprocessor system. Multistage Interconnection Networks (MINs) [1-10] are highly suitable for communication among tightly coupled nodes. For ensuring high reliability in complex systems, fault tolerance is an important issue. The basic idea for fault tolerance is to provide multiple paths for a source-destination pair, so that alternate paths can be used in case of a fault in a path [1-23]. However, to guarantee 1 -fault tolerance, a network should have a pair of alternate paths for every source destination pair which are disjoint in nature [1-8, 24-31].

Now-a-days applications of MINs are widely used for on-Chip communication. In past number of techniques has been used to increase the reliability and fault-tolerance of MINs, a survey of the fault-tolerance attributes of these networks is found in [1-6]. The modest cost of unique paths MINs makes them attractive for large multiprocessors systems, but their lack of fault-tolerance, is a major drawback. To mitigate this problem, three hardware options are available [1-5, 20-23]:

1. Replicate the entire network,

2. Add extra stages,

3. And /or Add chaining links.

4. Rearranging of the connection patterns with the addition or deletion of hardware links.

In addition to this, MINs can be designed to achieve fault tolerance and collision solving by providing a set of disjoint paths. Many researchers have done sufficient work on providing 1-fault tolerance to the MINs however; little attention has been paid to design the 3-Disjoint Paths Fault-tolerant MINs. We have been inspired by the work presented by the authors in [2431].

A Multi-stage interconnection network is fully able to meet the reliability demands if it is at least one fault tolerant that is there is at least one alternative path to deal with faults or collisions. This alternative path should be disjoint in nature with the existing routing path followed so that there is no such implication that if a switch or a link fails in the existing routing path then the alternative path will also fail. Most design of Multi-stage interconnection networks do not generate at least two disjoint paths and hence are not always fault tolerant resulting in packet losses and eventual performance degradation. Hence, this approach of two disjoint paths will always guarantee a way out of the problem of faults or collisions in a network [3234].

Whenever we want to design a interconnection network, we used to design them manually using the windows word and then hardwired them through the programming. At present, we do not have any tool through which we can develop the interconnection networks tool or this remains out of limelight therefore in this paper; we have discussed a tool designed for developing faulttolerant multi-stage interconnection networks. The designed tool is one of its own kind and will help the user in developing 2 and 3-disjoint path networks.

The rest of the paper is as follows: Section 2 discusses the testbed and experimental setup, new modules added to the existing case tool [32-34] and algorithm supported by the screen shots and the pseudocode followed by the conclusion and references.

\section{CASE TOOL: FAST INTERCONNECTIONS}

\subsection{Testbed and Experimental Setup}

CASE stands for "Computer Assisted Software Engineering. A CASE tool is a software tool that helps software designers and developers specify, generate and maintain some or all of the software components of an application. Many popular CASE tools provide functions to allow developers to draw database 
schemas and to generate the corresponding code in a data description language (DDL). Other CASE tools support the analysis and design phases of software development, for example by allowing the software developer to draw different types of UML diagrams [35].

We have designed both the networks using the Fast Interconnections tool and the architectural design of the software is already published in [33-34]. We have used Eclipse, is a multi-language software development environment comprising an integrated development environment (IDE) and an extensible plug-in system. It is written mostly in Java and can be used to develop applications in Java and, by means of various plug-ins, other programming languages. The IDE is often called Eclipse JDT for Java (i.e. JDK 1.6) and IDE is running on top of the IBM System $x$, running with Novell's SUSE Linux Enterprise Server 11. We have used advanced java features to build our system. The most important part of the tool is designing of the components, which are used to design disjoint paths MINs. We have design them in paint and stored them in component library. We have provided the access of this component within the tool using ComponentChooser class.

\subsection{New Module added to the Case Tool}

1. Added a new 3-Disjoint Paths Multi-stage Interconnection Network Simulator,

2. Design a circuit, enter a custom path, faulty component numbers, and click on simulate button,

3. Simulation will start and the path will turn green/red, depending on the packet drop with the faulty component marked by red cross every time a packet is dropped.

\subsection{Algorithm}

Algorithmic Step 1: Get path in A.

Algorithmic Step 2: Extract individual wire numbers in path1[].

Algorithmic Step 3: Get faulty_components in B.

Algorithmic Step 4: Extract individual comp. numbers in cmp1[].

Algorithmic Step 5: Verify the correctness of the path1[] and cmp1[].

Algorithmic Step 6: For 15 seconds repeats steps 7 and 8.

Algorithmic Step 7: For each wire in path1[] display it as green color for packet transfer.

Algorithmic Step 8: For every alternate packet drop a packet at components in cmp1[], display a red cross over components in cmp1[] and wires in path1[] as red color.

Algorithmic Step 9: End Simulation.

\subsection{Case Tool: Screen Shots}
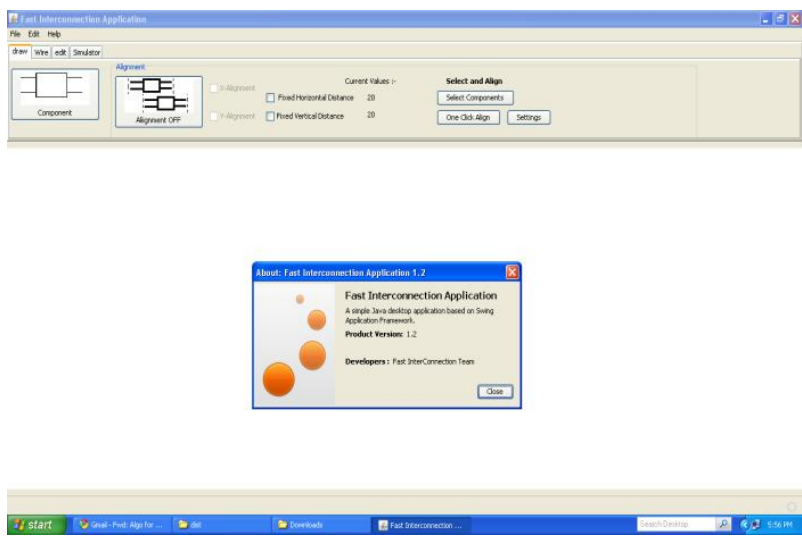

Fig 1: The front end of the Case Tool with the Welcome Message from the Fast Interconnections Group.

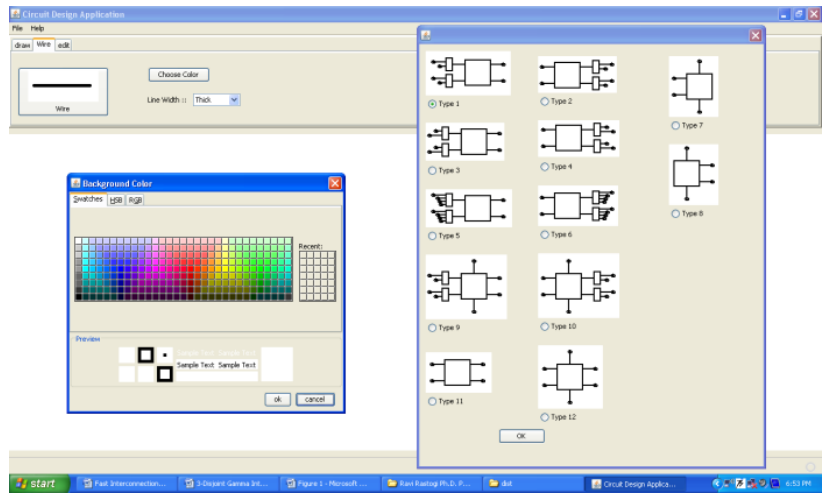

Fig 2: The front window with different widths of the wires, MIN Components and Color Chooser Applet.

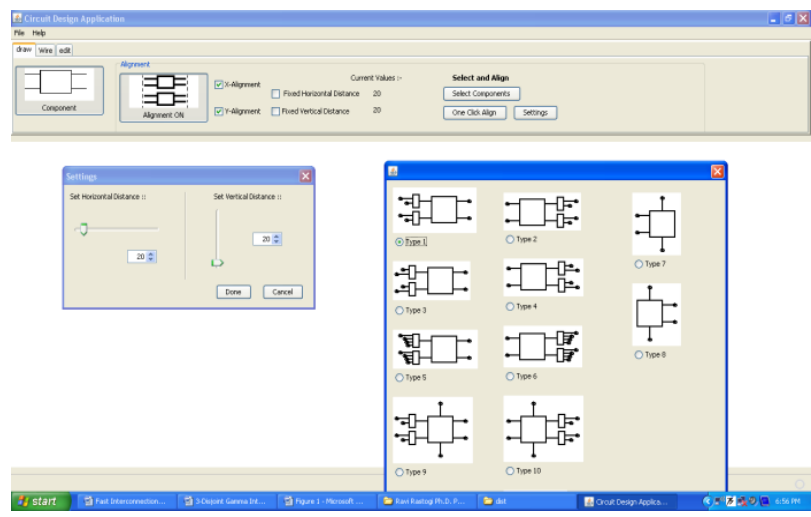

Fig 3: A case tool with various components and size of the application window can be fixed in terms of horizontal and vertical distance. 


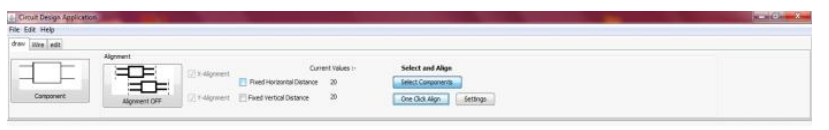

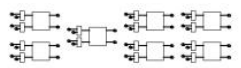

Fig 4: Shows that the elements have been aligned.

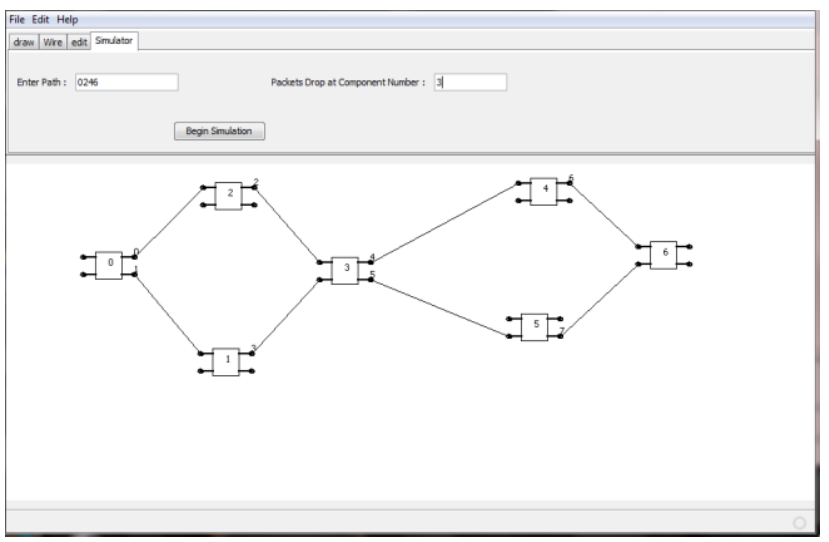

Fig 5: Shows that the components drawn using the draw method. We have changed the draw method which we have presented last time because of the addition of the new module.

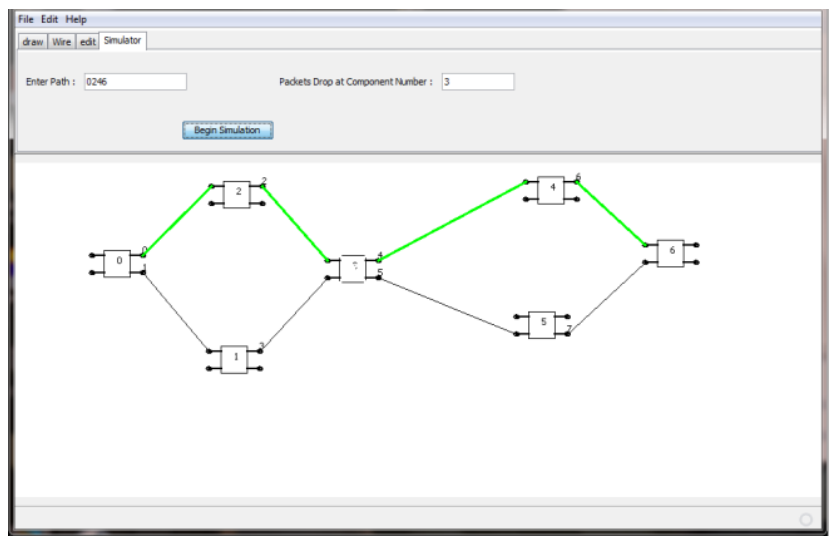

Fig 6: Highlighting the path in the MIN.

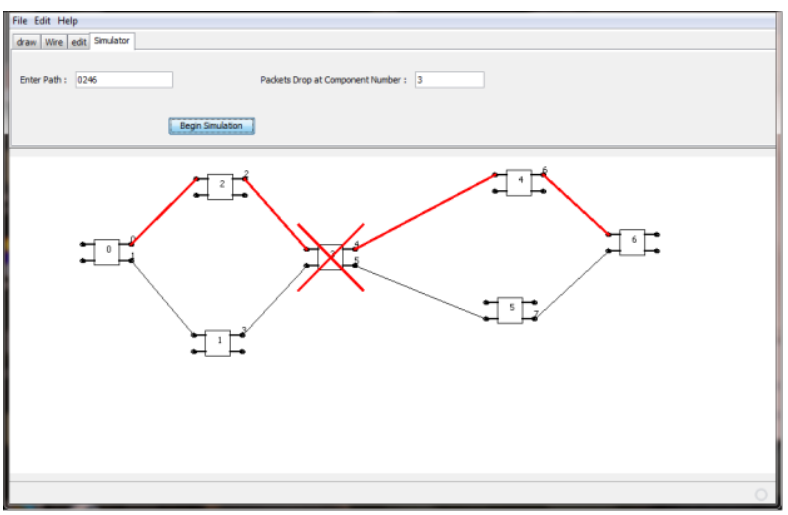

Fig 7: MIN with one faulty Component and dropping of Packet.

\subsection{Code for the New Module}

aAction

public Task simulate() throws Exception \{

sim_paint();

return new SimulateTask(getApplication());

\}

public void load_sim(File file) throws Exception \{

this.path $=$ file.getPath ()$;$;

System.out.println(this.path);

ois = new objectInputStream (new

FileInputStream(this.path));

for(int $i=0 ; i<100 ; i++)$

\{

$\operatorname{cmp}[i]=(\mathrm{cmp} 1)$ ois.readobject () ;

lc $[i]=$ (Color) ois.readobject () ;

thick $[i]=$ (Integer) ois.readobject () ;

line $[i]=$ (Integer) ois.readobject () ;

for (int $i=0 ; i<100 ; i++)$

\{

for (int $j=0 ; j<4 ; j++)$

\{

line [i] [j] = (Integer) ois.readobject ();

no_cmp $=$ (Integer) ois.readobject () ;

no line $=$ (Integer) ois.readobject () ;

ois.close ();

redraw ();

void simpaint()

\{

int $\mathrm{pkt}=0$;

Point p1,p2,p3,p4;

Graphics2D g=(Graphics2D)

canvas1.getGraphics ();

g.setStroke (new BasicStroke()) ;

g.setColor (Color.BLACK) ;

int $\mathrm{x}=0$;

String a=jTextFieldl.getText();

int [] pathl=new int [a.length()] ;

for (int $i=0 ; i<a$.length (); $i++)$ 
\{

path1 [i]=Character.getNumericValue (a.charAt (i)); \}

String $\mathrm{b}=j$ TextField2.getText();

int [] cmpl=new int [b.length()] ;

for (int $i=0 ; i<b$. length ()$; i++)$

\{

cmp1 [i] =Character. getNumericValue (b. charAt (i)) ;

\}

int $\mathrm{flag} 1=0$;

for (int $i=0 ; i<a$.length (); $i++)$

\{

if (path1 [i]>no_line)

\{

$f \operatorname{lag} 1=1 ;$

break;

\}

\}

int $\mathrm{flag} 2=0$;

for (int $i=0 ; i<b$. length () $i++)$

\{

if ( $\mathrm{cmp} 1[\mathrm{i}]>\mathrm{no}$ _cmp)

\{

flag2=1;

break;

\}

\}

int time $=5000000$;

if $(f \operatorname{lag} 1==1 \quad|| f \operatorname{lag} 2==1)$

\{

time=0;

if $(f \operatorname{lag} 1==1)$

JoptionPane.showMessageDialog (this.canvas1,

"Invalid Path. Please check the input.");

else if $(\mathrm{flag} 2==1)$

JoptionPane.showMessageDialog (this.canvas1,

"Invalid Component number. Please check the

input.");

\}

for(int $i=0 ; i<t i m e ; i++)$

\{

int $j$;

if (io(time/125)<24000)

\{

g.setColor (Color.RED);

for $(j=0 ; j<b$. length ()$; j++)$

\{

p3 $=$ cmp $[\mathrm{cmp} 1[j]]$. centre;

pkt++;

g.drawLine (p3.x-40, p3.y-40, p3.x+40,p3.y+40);

g.drawLine (p3.x-40, p3.y+40, p3.x+40,p3.y-40); \}

\}

else

\{

g.setColor (g.getBackground ()) ;

for $(j=0 ; j<b$. length ()$; j++)$

\{

p3 $=$ cmp $[\mathrm{cmp} 1[j]]$. centre;

g.drawLine (p3.x-40, p3.y-40, p3.x+40,p3.y+40);

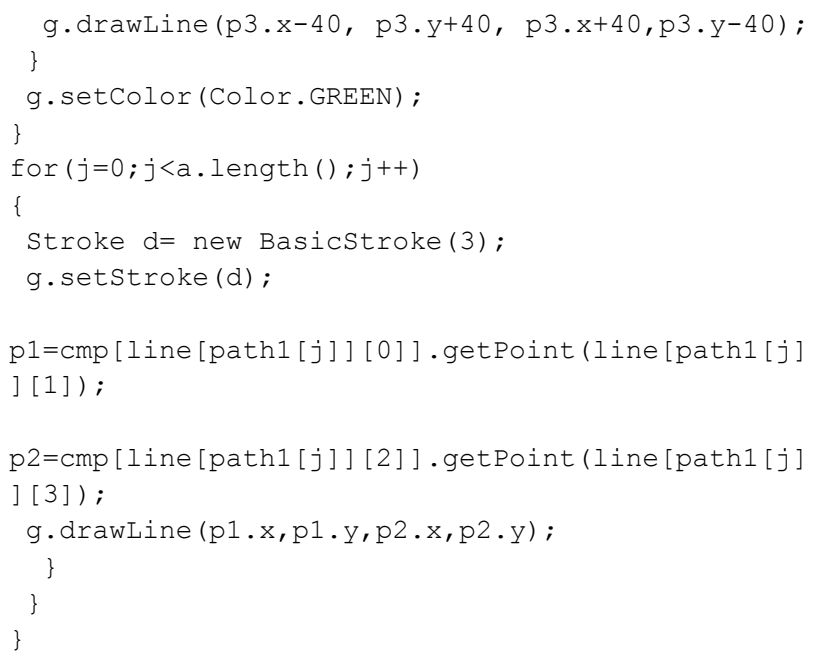




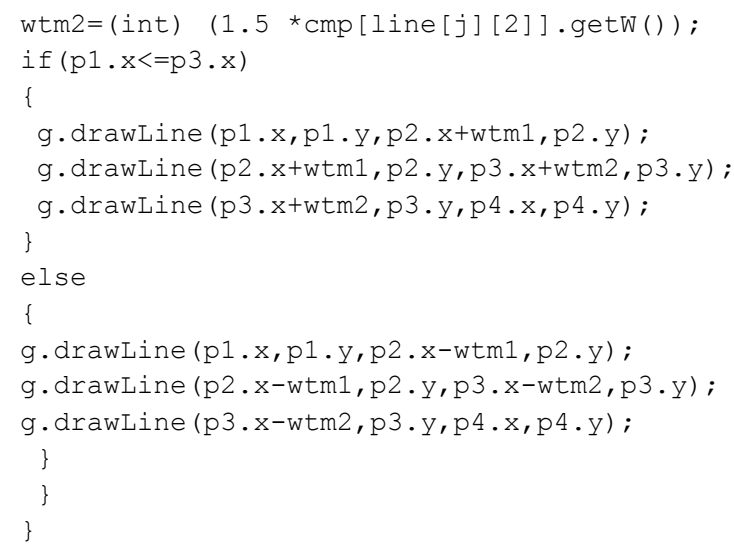

\section{CONCLUSION AND FUTURE WORK}

In this paper, we have discussed a the newly added module to the existing tool called as Fast Interconnections, which have been designed to develop the 2 and 3-disjoint path multi-stage interconnection network. We have provided the algorithm of the new simulator supported by the screen shots and pseudocode.

The current of the newly added module are as followssimulation run time and the amount of packets dropped is currently fixed, faulty components are to be input before simulation starts. Further work- we will maintain a database for dropped packets, and allow user to dynamically drop packets from anywhere in the circuit during simulation.

\section{REFERENCES}

[1] T.Y. Feng, A survey of interconnection networks, IEEE Computer 14, pp. 12-27, 1981.

[2] G.B. Adams III, D.P. Agrawal and H.J. Siegel, A survey and comparison of fault-tolerant multi-stage interconnection networks, IEEE Computer 20, pp. 14-27, 1987.

[3] W.J. D`ally, Scalable Switching Fabrics for Internet Routers, White paper, Avici Systems Incorporation, 2001.

[4] L.N. Bhuyan, Special issue of interconnection networks, IEEE Computer, Vol. 20 (6), June 1987.

[5] H.J. Siegel, Interconnection Network for Large Scale Parallel Processing: Theory and Case Studies, McGraw Hill, ISBN 0-07-057561-4, 1990.

[6] K. Hwang, Advanced Computer Architecture: Parallelism, Scalability, Programmability, Tata McGraw-Hill, India, ISBN 0-07-053070-X, 2000.

[7] J. Duato, S. Yalamanchili and L.M. Ni, Interconnection Networks: An Engineering Approach, Morgan Kaufmann, ISBN 1-55860-852-4, 2003.

[8] W.J. Dally and B. Towles, Principles and Practices of Interconnection Networks, Morgan Kaufmann, San Francisco, ISBN 978-0-12-200751-4, 2004.

[9] H.R. Arabnia and M.A. Oliver, Arbitrary Rotation of Raster Images with SIMD Machine Architectures, International Journal of Eurographics Association (Computer Graphics Forum), 6(1), pp. 3-12, 1987.
[10] S.M. Bhandarkar, H.R. Arabnia and J.W. Smith, A Reconfigurable Architecture For Image Processing And Computer Vision, International Journal of Pattern Recognition And Artificial Intelligence, 9(2), pp. 201-229, 1995.

[11] S.M. Bhandarkar and H.R. Arabnia, The Hough Transform on a Reconfigurable Multi-Ring Network, Journal of Parallel and Distributed Computing, 24(1), pp. 107-114, 1995.

[12] M.A. Wani and H.R. Arabnia, Parallel Edge-Region-Based Segmentation Algorithm Targeted at Reconfigurable MultiRing Network, The Journal of Supercomputing, 25(1), pp. 43-63, 2003.

[13] J. Duato, A New Theory of Deadlock-free Adaptive Routing in Wormhole Networks, IEEE Transactions on Parallel and Distributed Systems, 4(12), pp. 1320$1331,1993$.

[14] J. Duato, A Necessary and Sufficient Condition for Dead lock-free Adaptive Routing in Wormhole Networks, IEEE Transactions on Parallel and Distributed Systems, 6(10), pp. 1055-1067,1995.

[15] W.J. Dally and C.L. Seitz, Deadlock-Free Message Routing in Multiprocessor Interconnection Networks, IEEE Transactions on Computers, C-36(5), pp. 547-553, 1987.

[16] W.J. Dally and H. Aoki, Deadlock-Free Adaptive Routing in Multi computer Networks Using Virtual Channels, IEEE Transactions on Parallel Distributed Systems, 4(4), 1993.

[17] J. Duato, Deadlock-Free Adaptive Routing Algorithms for the 3DTorus: Limitations and Solutions, In Proceedings of Parallel Architectures and Languages Europe 93, 1993.

[18] Nitin and A. Subramanian, Efficient Algorithms to Solve Dynamic MINs Stability Problems using Stable Matching with Complete TIES, Journal of Discrete Algorithms, 6(3), pp. 353-380, 2008.

[19] A. Singh, W.J. Dally, A.K. Gupta, B. Towels, Adaptive Channel Queue Routing on k-ary n-cubes, Proceedings of the sixteenth annual ACM symposium on Parallelism in algorithms and architectures, 2004.

[20] Nitin, S. Garhwal and N. Srivastava, Designing a Faulttolerant Fully-chained Combining Switches Multi-stage Interconnection Network with Disjoint Paths, The Journal of Supercomputing, DOI 10.1007/s11227-009-0336-z, pp. 1-32, 2009

[21] Nitin and D.S. Chauhan, Comparative Analysis of Traffic Patterns on k-ary n-tree using Adaptive Algorithms based on Burton Normal Form, Journal of Supercomputing, DOI: 10.1007/s11227-010-0454-7, pp. 1-20, 2010.

[22] Nitin, V.K. Sehgal and P.K. Bansal, On MTTF analysis of a Fault-tolerant Hybrid MINs, WSEAS Transactions on Computer Research, ISSN 1991-8755, 2(2), pp. 130-138, 2007.

[23] Nitin, Component Level Reliability analysis of Faulttolerant Hybrid MINs, WSEAS Transactions on Computers, ISSN 1109-2750, 5(9), pp. 1851-1859, 2006. 
[24] C.W. Chen and C.P. Chung, Designing a disjoint path interconnection network with collision solving and fault tolerance, The Journal of Supercomputing, 34(1), pp. 63$80,2005$.

[25] C.W. Chen, Design schemes of dynamic rerouting networks with destination tag routing for tolerating faults and preventing collisions, The Journal of Supercomputing, 38(3), pp. 307-326, 2006.

[26] H.J. Siegal, D.R. Jose and A.B. Fortes, Destination tag routing techniques based on a state model for the IADM network, IEEE Transaction on Computers, 41(3), pp. 274$285,1992$.

[27] B. Smith, Design of dynamic rerouting networks with destination tag routing for tolerating faults and preventing collisions, Springer Science, 2006.

[28] D.S. Parker and C.S. Raghavendra, The gamma network, IEEE Transactions on Computers, 33, pp. 367-373, 1984.

[29] P.J. Chuang, CGIN: A fault tolerant modified gamma interconnection network, IEEE Transactions on Parallel and Distributed Systems, 7(12), pp. 1301-1306, 1996.

[30] C.W. Chen, N.P. Lu, T.F. Chen, and C.P. Chung, Faulttolerant gamma interconnection networks by chaining, In
IEE Proceedings on Computers and Digital Techniques, 147(2), pp. 75-80, 2000.

[31] C.W. Chen, N.P. Lu, and C.P. Chung, 3-Disjoint gamma interconnection networks, The Journal of Systems and Software, 66, pp .129-134, 2003.

[32] R. Rastogi, Nitin and D.S. Chauhan, 3-Disjoint Paths Faulttolerant Omega Multi-stage Interconnection Network with Reachable Sets and Coloring Scheme, Proceedings of the 13th IEEE International conference on Computer Modeling and Simulation (IEEE UKSim), Emmanuel College, Cambridge, UK, March 30-April 1, 2011.

[33] R. Rastogi, Nitin and D.S. Chauhan, Fast Interconnections: A Case Tool for Developing Fault-tolerant Multi-stage Interconnection Networks, International Journal of Advancements in Computing Technology, ISSN: 20058039, 2(5), December 2010, pp. 13-24.

[34] R. Rastogi and Nitin, On a Fast Interconnections, International Journal of Computer Science and Network Security, ISSN: 1738-7906, 10(8), August 2010, pp. 74-79.

[35] ERCIM Working Group Software Evolution, available at http://wiki.ercim.eu/wg/SoftwareEvolution/index.php/Term inology 